Brit. J. industr. Med., 1953, 10, 93.

\title{
DUST SAMPLING TO SIMULATE THE HUMAN LUNG
}

\author{
BY \\ H. H. WATSON \\ From the Pneumoconiosis Research Unit of the Medical Research Council, \\ Llandough Hospital, Penarth, Glam.
}

(RECEIVED FOR PUBLICATION SEPTEMBER 2, 1952)

There are many difficulties associated with sampling an air-borne dust and with assessing its concentration, especially when the potential danger to the lungs is being considered. This is evident from the fact that such a large number of different dust-sampling instruments have been devised from time to time, no two of which give the same results under all conditions. This multiplicity of instruments is due partly to the fact that there is no fundamental agreement on the appropriate index of concentration to represent the pneumoconiosis hazard of a dust ; whether it should be in terms of particle number, surface area, or mass. The relationships between these different units depend very much on the size-distribution of the particles in the dust being considered: different instruments sample particles of different sizes with varying efficiencies. Until recently there has not been any satisfactory quantitative information on the manner in which dust particles penetrate as far as the lung alveoli.

It is the purpose of this paper to consider the effect of certain physical parameters of dust particles, e.g. size, density, and shape, on lung deposition. Subsequently, an examination is made of the possibilities of taking dust samples that are representative of the proportional deposition of the different sizes in the lung. Consideration is given to the different units in which concentration may be expressed. In many dust clouds most of the mass occurs in particles too large to penetrate to the lung alveoli. Table 1 gives the size-distribution of a typical coal-dust cloud; the area and mass distributions have been calculated from the number distribution determined with a microscope.

This is a coarse dust from the point of view of the lung. When the size distributions of the dust, the shape, and density of particles are taken into account (see Fig. 5) the lung, i.e., alveolar, deposition has been calculated to be :-

C

$\begin{array}{lll}\text { By number } & \ldots & 30 \% \\ \text { By area } & \ldots & 8.4 \% \\ \text { By mass } & \ldots & 3.0 \%\end{array}$

As would be expected, the amount of dust deposited in the lung from a dusty environment depends, among other things, on the size-distribution of the dust. The problem becomes, therefore, how to develop an instrument which selects dust particles of different sizes, densities, and shapes in the same proportion as they are deposited in the lung, so to give an estimate of the respirable dust.

The best information available on alveolar deposition is probably that given by Brown, Cook, Ney, and Hatch (1950). They made over 100 tests with human subjects and sampled the dust breathed in and out through the mouth. Respiration rate was controlled with a Drinker respirator to 15 a minute, the volume per breath varying from 600 to $800 \mathrm{ml}$. The exhaled dust was partitioned into a number of fractions so that the alveolar fraction could be separated from that coming from different parts of the upper respiratory system. Other investigators have not made this refinement, and with the exception of Wilson and LaMer (1948) have not been able to report more than the retention

TABLE 1

PERCENTAGE DISTRIBUTIONS OF PARTICLE NUMBER SURFACE AREA, AND MASS WITH PARTICLE "DIAMETER" FOR A TYPICAL SOUTH WALES COAL-MINE DUST CLOUD

\begin{tabular}{c|c|c|c}
\hline $\begin{array}{c}\text { Size Group } \\
(\mu)\end{array}$ & Number & $\begin{array}{c}\text { Surface } \\
\text { Area }\end{array}$ & Mass \\
\hline$<1^{*}$ & 28.9 & $0 \cdot 7$ & - \\
$1-2$ & 21.0 & 3.3 & 0.5 \\
$2-4$ & 34.6 & $19 \cdot 8$ & $4 \cdot 7$ \\
$4-8$ & 11.9 & 30.1 & 12.8 \\
$8-16$ & 3.0 & 26.6 & 28.0 \\
$16-32$ & 0.575 & 12.9 & 26.0 \\
$32-64$ & 0.025 & $5 \cdot 7$ & 21.0 \\
$>64$ & 0.005 & 0.9 & 7.0 \\
\hline
\end{tabular}

*Size of particle is given in terms of mean projected diameter. The area and mass distributions have been computed on the assumption that there is no statistical change of shape of particle with size. The dust code number is A(i) of Table 3. 


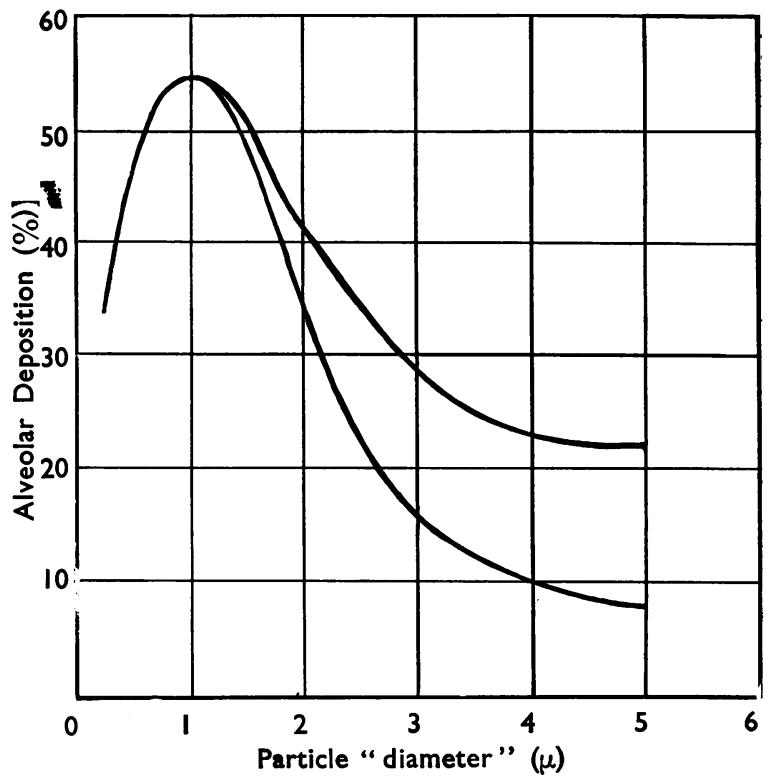

FIG. 1.-Lung deposition curves (Brown, Cook, Ney, and Hatch, 1950) china clay particles $(\rho=2 \cdot 60)$.

of the respiratory system as a whole, or of the nose separately. Wilson and LaMer estimated deposition in the alveoli and lower air passages by direct activity counts on the chest, using radioactive dusts of known size.

Brown and others derived from their measurements the two full curves shown in Fig. 1. The upper curve was calculated on the assumption that upper-respiratory deposition was equal in both directions ; theolower curve is based on an assumed one way upper-respiratory retention. The authors consider the true relationship to lie somewhere between the two. It will be noticed that the curves do not extend to particles smaller than $0.25 \mu$ or larger than $5 \mu$ diameter, and that they tend to flatten out in the approach to the larger sizes. Davies $(1949,1952)$ in reviewing the literature has stated that spherical particles of unit density larger than $12 \mu$ are completely retained in the nose. Accepting this value for the present purpose, we can say that nothing larger than this will penetrate to the alveoli, but that smaller particles do stand a chance of doing so. It is probable that even with mouth breathing the maximum size penetrating as far as the alveoli remains much the same.

\section{Physical Parameters of Dust Particles}

In order to discuss quantitatively the deposition of dust and the significance of the curves shown in Fig. 1 (and so to derive design data for dust-sampling instruments to give directly a measure of hazard), it is necessary to consider something of the physical make-up of dust particles. It is usual to refer to the "diameter" of a particle as a characteristic of its size. The looseness of this custom is at once apparent from Fig. 2, which is a photomicrograph of air-borne coal dust. A particle of dust is anything but a sphere, and the microscope only gives us information concerning the projection of the particle on a single plane; the shape in the third dimension is not revealed. Frequently we see aggregates of particles, and these are clearly more difficult still to characterize by a "diameter" estimated from the microscopic appearance. It is necessary, therefore, to attempt to assess a characteristic of a particle, or an aggregate of particles, which bears a definite relationship to its motion in the free air or in the respiratory system. An appropriate measure is the aerodynamic or Stokes's diameter, which is the diameter of a sphere which falls in free air at the same rate as the particle; we can either consider the sphere to be of unit density or of that of the material of the particle, if this be known. Alternatively we could define the size of a particle directly in terms of its Stokes's terminal velocity. For a spherical particle this is given by :-

$$
\mathrm{V}_{\mathrm{s}}=\frac{\mathrm{d}^{2} \rho \mathrm{g}}{18 \eta}\left(1+\frac{\mathrm{AL}}{\mathrm{d}}\right)
$$

where $V_{s}=$ terminal velocity, $d=$ diameter of sphere,

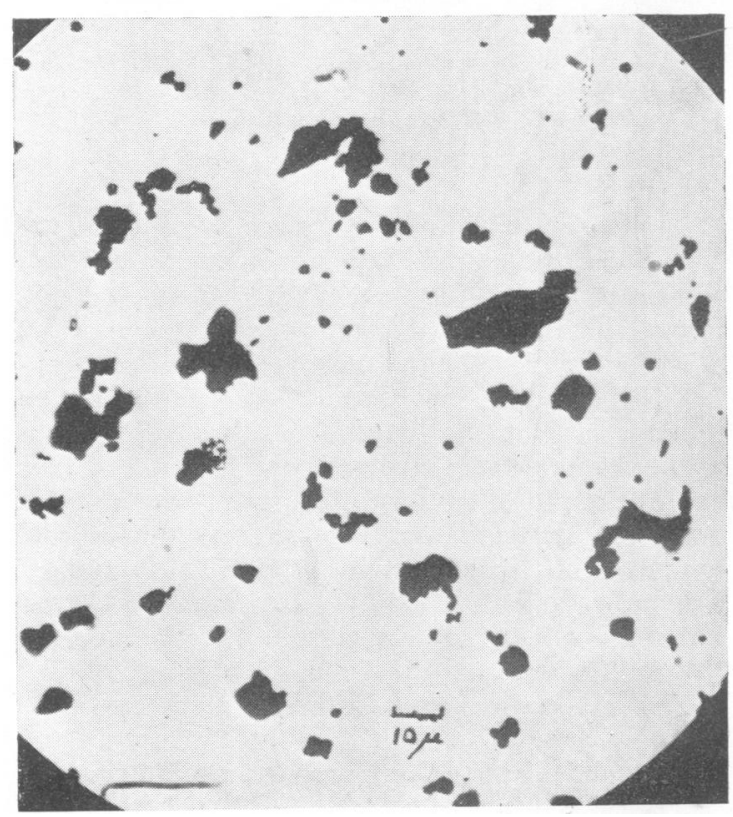

FIG. 2.-Photomicrograph of coal dust. 
$\rho=$ density of sphere less density of fluid through which sphere is falling, $\eta=$ viscosity of fluid, $\mathrm{g}=$ acceleration due to gravity, $\mathrm{A}=\mathrm{a}$ constant (about $1 \cdot 6$ ), $\mathrm{L}=$ mean free path of air molecules (about $10^{-5} \mathrm{~cm}$.).

This equation holds for spheres of unit density up to about $80 \mu$ diameter. The function in brackets is the Cunningham correction for slip through the discontinuities of the medium and is only significant for small particles, being some $8 \%$ for spheres of diameter $2 \mu$. Apart from the term in the Cunningham correction, we see that the property of the sphere that determines its terminal velocity in air is very nearly the product of the square of the diameter and its density.

The passage of air from the nose to the depths of the lung and back again is extremely tortuous. Particles in this air will lag behind any change in direction and will tend to be thrown out by centrifugal force and by impingement, as well as to be deposited under the effect of gravity. For all these processes, it is the same parameter, viz. $d^{2} \rho$, that determines the degree and rate of removal. In other words, the terminal velocity of a sphere, or indeed of a particle or aggregate of any shape, is the parameter that determines its behaviour in penetrating the respiratory system. Only for spheres can we assess this parameter by direct measurement under the microscope.

\section{Experimental Determination of Aerodynamic Diameter}

We are faced, therefore, with the problem of determining the aerodynamic diameters of real dust particles. An attempt has been made to do this directly using a conifuge. This is an instrument devised by Sawyer and Walton (1950), in which an indrawn cloud is "winnowed" in such a way that the particles are classified according to their terminal velocities. Dust enters the apex of the annular space between a rotating solid cone and a similarly rotating outer conical sheath on to which the particles are precipitated by centrifugal force. Large particles are deposited close to the entrance of the instrument, successively smaller sizes being deposited further away from the sampling orifice. When particles are of similar shape and density, the settling velocity is a unique function of size, and the deposit is in the form of a spectrum of particle sizes with a high degree of purity. This may be demonstrated by examining the deposit from a cloud of spherical particles. At any one position in the spectrum the deposit will consist of particles with the same terminal velocity, irrespective of the density and shape of the particle or of the complexity of an aggregate.
Dusts were rendered air-borne in the laboratory and sampled with the conifuge. Similarly, small spheres of pyrex glass $(0=2.25 \mathrm{~g}$. $/ \mathrm{cc}$.) were sampled on the same slide as the dust particles. The mean projected diameter $\left(d_{p}\right)$ of a large number of particles was measured, and corresponding to each dust particle the diameter of a neighbouring sphere on the deposit was also measured. Allowing for the respective densities of particle and sphere the value of the aerodynamic diameter, $d_{s}$, for each particle was calculated. Only discrete or unitary particles have so far been considered.

The conifuge has been used in this way to determine the ratio $d_{p}: d_{s}$ for a number of different dusts. Full details of this work will be published elsewhere and only the results obtained for coaldusts and for china-clay and quartz are given here (Table 2). Measurements were made on particles

TABLE 2

RATIO OF MEAN PROJECTED DIAMETER TO AERODYNAMIC DIAMETER FOR A NUMBER OF COAL DUSTS AND FOR CHINA-CLAY AND QUARTZ DUST

\begin{tabular}{lll|c|c|c}
\hline \multicolumn{2}{c|}{ Origin of Dust } & $\begin{array}{c}\text { Value of } \\
\text { Mean } \\
\mathrm{d}_{\mathrm{p} / \mathrm{d}}\end{array}$ & $\begin{array}{c}\text { Standard } \\
\text { Error of } \\
\text { Mean }\end{array}$ & $\begin{array}{c}\text { No. of } \\
\text { Particles } \\
\text { Measured }\end{array}$ \\
\hline S. Wales & $\ldots$ & $\ldots$ & 1.35 & 0.015 & 128 \\
Lancashire & $\cdots$ & $\cdots$ & 1.31 & 0.013 & 124 \\
Cumberland & $\cdots$ & $\cdots$ & 1.35 & 0.012 & 147 \\
\hline China clay & $\ldots$ & $\ldots$ & 1.61 & 0.016 & 134 \\
\hline Quartz.. & $\cdots$ & $\cdots$ & 1.67 & 0.021 & 150 \\
\hline
\end{tabular}

over a range of $\mathrm{u}_{\mathrm{p}}$ of from $2 \mu$ to $8 \mu$, and within this range there was no indication of any change in the ratio with particle size (Fig. 3).

\section{Derivation of Standardized Lung-Deposition Curve}

Measurements of the aerodynamic diameters were made for china-clay, because Brown and his colleagues used dust of this material for their lungdeposition experiments, in which they estimated particle size in terms of the Feret statistical diameter. Measurements were made of the ratio of the Feret diameter to the mean projected diameter for the sample of china clay dust examined, the mean value being found to be 1.15 (standard error 0.01). We can, using this ratio, correct the particle size of Fig. 1 to mean projected diameter, but the resulting curves will be appropriate only for china-clay dust, with particle size measured in terms of $d_{p}$, the mean projected diameter. Other dusts contain particles of different density and different mean $d_{p} / d_{s}$, and it is possible to construct curves for individual dusts. As an intermediate stage in the necessary computation, a standardized lung-deposition curve for spheres of unit density has been 


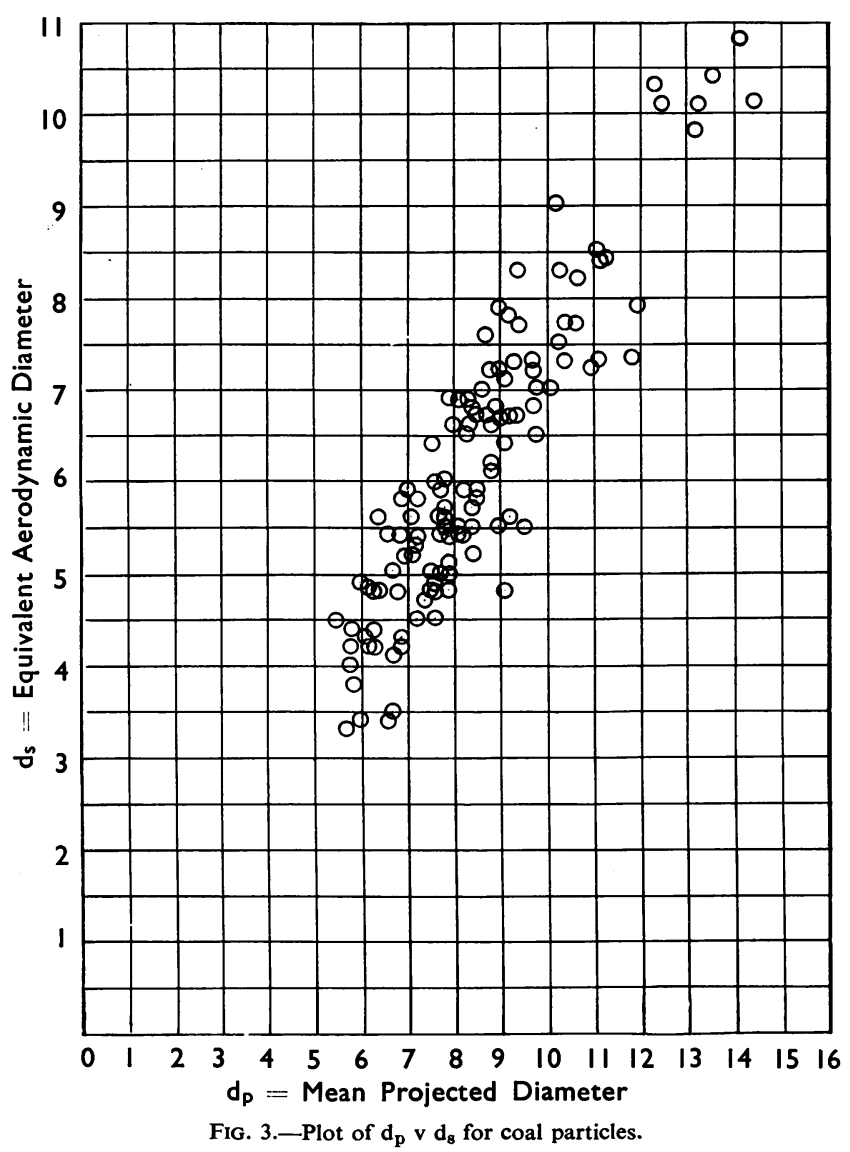

(Davies, 1949). It is mainly because the amended lower curve of Fig. 1 extrapolates smoothly to zero deposition at $12 \mu$ diameter that it has been selected in preference to the upper curve or any intermediate curve. The actual shape and position of the extrapolated part of the curve is of little importance for the argument developed in this paper. The absolute values of the ratios given in Table 4 are dependent somewhat on the manner of the extrapolation, but not so their relative values.

Using the standardized lung-deposition curve we can work in the reverse direction and draw up curves relating to any dust, for which the density and the mean $d_{p} / d_{s}$ are known. Taking the density of coal to be $1.31 \mathrm{~g}$. $/ \mathrm{cc}$., the determined mean value for the samples examined and that of quartz to be $2.65 \mathrm{~g}$./cc., Figs. 5 and 6 have been constructed for these two substances.

These lung-deposition curves can be used to estimate the fraction of a dust cloud that will be deposited in an arbitrary "standard lung" operating under the conditions of the experiments of Brown and others. The lung deposition curves can only be applied directly to calculations for aggregate-free dusts, but an aggregate will stand the same relative chance, depending on its aerodynamic diameter,

constructed (Fig. 4). This has been derived from the lower curve of Fig. 1, the particle size scale having been amended by the following steps :-

(1) Divide given diameter scale by $1 \cdot 15$ to convert from Feret diameter to mean projected diameter; (2) divide by 1.61 the mean value of $d_{p} / d_{s}$ for china-clay dust ; (3) multiply by $\sqrt{2 \cdot 60}$, where $2 \cdot 60$ is the density of the china clay dust. This is done because the terminal velocity of a particle is proportional to the product of density and the diameter squared, and so the aerodynamic diameter of a sphere of unit density is larger than that of a particle of density $\rho$ by a factor equal to the square root of $\rho$.

The derived curve of Fig. 4 has been smoothly extrapolated to zero deposition at $12 \mu$ diameter, the minimum size of sphere of unit density that is completely retained by the nose

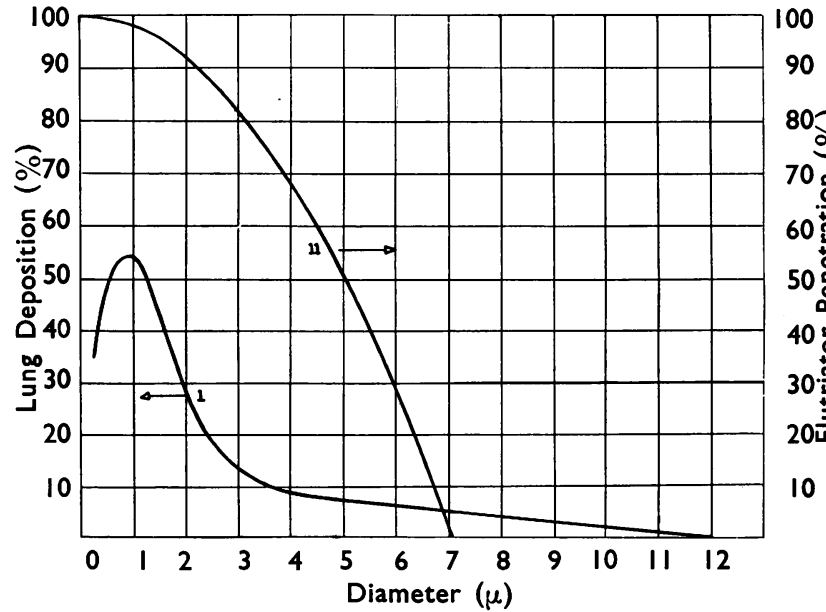

FIG. 4.-I. Standardized lung deposition curve for spheres of unit density.

II. Standard elutriator penetration curve for spheres of unit density. 
of penetrating a selector device (see below) as an equivalent unitary particle or sphere.

\section{Dust-Sampling Instruments and Lung Deposition Curves}

We shall now consider the relationships between the performance of certain sampling instruments and the standardized lung deposition curve of Fig. 4, or of its derivatives, Figs. 5 and 6 . An ideal instrument should reject everything larger than the aerodynamic equivalent of a $12 \mu$ diameter sphere of unit density, and select smaller particles in proportions, depending on their sizes, as indicated by the curves. As any selector device in a real instrument will of necessity be much simpler in construction than the human respiratory system, it is likely that only an approximation to any chosen lung deposition curve will be obtained. We are, however, really concerned only with the relative shapes of the selector penetration and lung deposition curves, as absolute values may be adjusted as required by instrumental constants.

Four possible methods of selecting a respirable fraction of dust will be considered : (1) elutriation, (2) impaction, (3) centrifugal force (cyclone), (4) selective counting of a thermal precipitator or similar samples collected on cover glasses.

With all these methods, large particles may be trapped readily or ignored (Method 4). That fraction of the dust passing the selective device constitutes the sample representative of what is likely to be deposited in the lung. It must of course be collected in a suitable manner on a filter or a glass surface, for subsequent assessment in an appropriate way.

Elutriation.-Walton (1950) has shown that both for the conventional vertical elutriator and for a

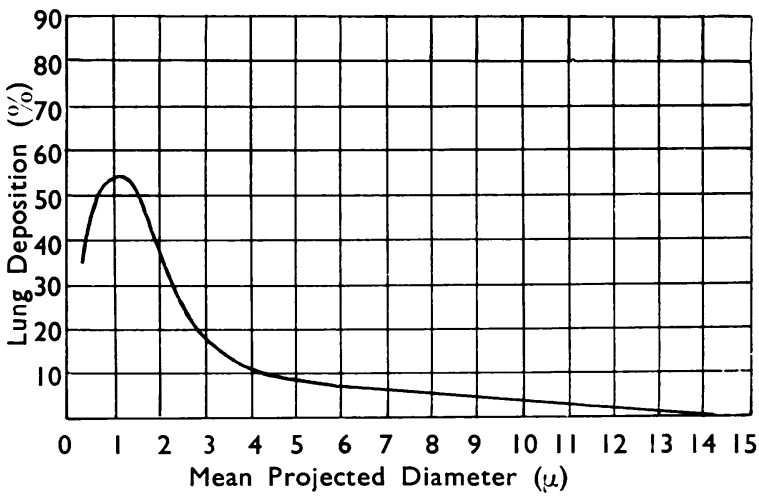

FIG. 5.-Standardized lung deposition curve for coal $\left(\rho=1 \cdot 3 \frac{d_{p}}{d_{s}}\right.$ $=1 \cdot 35)$. horizontal plate separator through which dusty air is passed particles will penetrate according to equation (2)

$$
\mathrm{P}=\left(1-\frac{\mathrm{ds}^{2}}{\mathrm{do}^{2}}\right) \quad \text {. . . . . . }
$$

where $\mathbf{P}$ is the probability of a particle with aerodynamic diameter $d_{s}$ passing and being collected as part of the sample, $d_{o}$ being the aerodynamic diameter of the cut-off size, i.e., the size which just does not penetrate and which depends on the design of the apparatus. This property of separation has been suggested by Walton (1950) for the characteristics of an ideal sampling instrument, with $50 \%$ sampling, i.e., penetration through the separator, for spheres $5 \mu$ diameter of unit density. The resulting curve is shown in Fig. 4, and may be compared with the standardized lung-retention curve derived from the experimental results of Brown and others (1950).

Impaction.-An impactor device could be designed to give the same type of selection, or one with an even sharper degree of separation. The practical difficulties of this particular approach are rather large and impactors will not be considered further.

Centrifugal Separation.-A small cyclone separator appears to allow a penetration of dust more closely resembling penetration to the alveoli than does an elutriator device. In particular, the curve relating penetration to particle size is concave upwards (Linden, 1949) similar to that of the main part of the lung-retention curve, whereas that for the elutriator is convex upwards. In Fig. 7 are given the results obtained on the penetration through a small cyclone of two different coal-dust clouds. The first was a cloud produced in the laboratory which contained very few aggregates, the second a

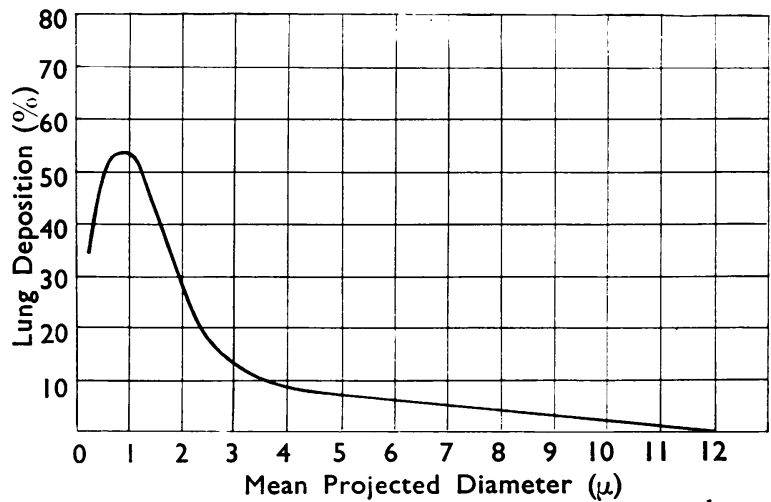

Fig. 6.-Standardized lung deposition curve for quartz $\left(\rho=2.65 \frac{d_{\mathbf{p}}}{d}\right.$. $=1.67$ ) 
coal-mine cloud containing its usual quota of aggregates.

The two fractions of the dust, that retained by the cyclone and that passing which was collected on a suitable filter, were examined separately. Portions of each fraction were mounted in a dispersed state in Canada balsam and number size-distributions were made under a microscope. From these the corresponding mass-distributions were calculated, and knowing the relative masses of dust of all sizes retained in and passing the cyclone, the actual curve of retention against size was developed. The results for the two dusts are given in Fig. 7 in terms of mean projected diameter, together with the theoretical elutriator curve for coal (dotted line) also in terms of the mean projected diameter. These curves may be compared with the derived-lung deposition curve for coal (Fig. 5).

The two cyclone curves differ appreciably, and the reason probably lies in the different states of aggregation of the two dusts. The laboratory dust contained very few aggregates and the experimentally determined penetration curve corresponded well with that to be expected, with penetration tending to $100 \%$ for the smaller particles.

Aggregates present in the dusts would be broken down when representative fractions were mounted in Canada balsam for microscopical ex̧amination. In particular, small particles attached to larger ones would be stopped in the cyclone and so included in the retained fraction and seen and counted as small particles. Had such particles not been part of a relatively heavy aggregate, they would have penetrated. It has been thought that many aggregates are broken down in a cyclone, but the present results suggest that, on the contrary, further aggregation may take place.

The cyclone penetration curve No. 1 , which is for the non-aggregated dust, and not curve No. 2 , is suitable for direct comparison with the curves given in Figs. 4, 5, and 6.

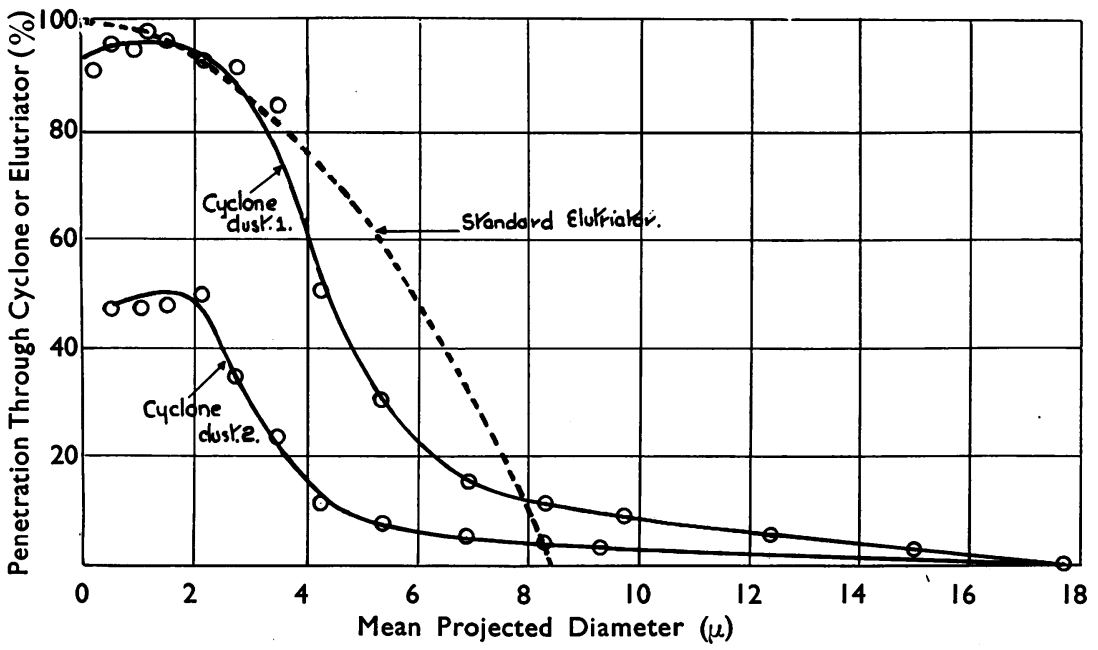

Selective Counting.-The fourth possibility is to count over a restricted range of sizes the particles in a thermal precipitator or similar quantitative sample. This will be the equivalent of a sharp cut-off at the top and at the bottom of the selected range, $100 \%$ of the particles within the range being counted. In so far as the aerodynamic diameter of a particle and particularly that of an aggregate cannot be estimated directly by microscopical examination, this approach is not likely to be as satisfactory as the two others that have been discussed in some detail, which collect or reject an aggregate according to its aerodynamic diameter in the manner indicated by the appropriate curves. There are also errors involved in deciding under the microscope the correct $d_{p}$ of an irregularly shaped particle, and thus whether or not to include or exclude these particles near the chosen limits of size. Apart from this, microscopic assessment of dust is a tedious matter, and it can be argued that particle number concentration is not likely to be as good an index of hazard as area or mass concentration within the respirable range of size. On the other hand, if a selective device is used with a thermal precipitator, there are two possibilities of assessing quickly the resulting sample. One is to obtain a measure of the surface area of the dust by means of a photoelectric densitometer. The other is to count the deposit electronically using a suitable scanning device; developments on this line are reported by Walton (1952).

Performance of Selector Devices Compared with Lung.-The alveolar deposition of a number of dusts has been calculated in terms of particle number, surface, area, and mass, using the appropriate derived lungdeposition curves. In addition, for comparison, the penetration through two instruments, an elutriator or horizontal separator and the small cyclone (Dust 1), have also been calculated. To make results comparable, the lung depositions and instrument penetrations have been calculated in each case for 100 particles of the various dusts,

FIG. 7.-Dust penetration through small cyclone separator compared with theoretical penetration through standard elutriator (coal dust). 
counts being made down to about $0 \cdot 2 \mu$ with a $2 \mathrm{~mm}$. objective. Details of the dust examined are given in Table 3.

TABLE 3

NATURE AND SIZE-DISTRIBUTION OF DUSTS USED FOR CALCULATION OF LUNG DEPOSITION AND OF SELECTOR DEVICE PENETRATION

\begin{tabular}{|c|c|c|c|c|c|c|}
\hline \multirow[t]{2}{*}{ Dust } & \multirow{2}{*}{$\begin{array}{l}\text { Dust } \\
\text { Code } \\
\text { No. }\end{array}$} & \multirow[t]{2}{*}{$\begin{array}{l}\text { Sampling } \\
\text { Method }\end{array}$} & \multicolumn{4}{|c|}{$\begin{array}{l}\% \text { Occurrence by Number } \\
\text { in Size-range (mean } \\
\text { projected diameter in } \mu)\end{array}$} \\
\hline & & & $<0.5$ & $0 \cdot 5-2$ & $2-5$ & $>5$ \\
\hline $\begin{array}{l}\text { Coal-mine dusts } \\
\text { S. Wales } \\
\text { S. Wales } \\
\text { Cumberland } \\
\text { Lancashire .. }\end{array}$ & $\begin{array}{l}\text { A (i)* } \\
\text { A (ii) } \\
\text { A (iii) } \\
\text { A (iv) }\end{array}$ & $\begin{array}{l}\text { Filter } \\
\text {," }\end{array}$ & $\begin{array}{r}13 \\
7 \\
2 \\
1\end{array}$ & $\begin{array}{l}38 \\
45 \\
40 \\
31\end{array}$ & $\begin{array}{l}39 \\
37 \\
48 \\
52\end{array}$ & $\begin{array}{l}10 \\
11 \\
20 \\
16\end{array}$ \\
\hline $\begin{array}{l}\text { S. Wales } \\
\text { Cumberland } \\
\text { Cumberland } \\
\text { Cumberland }\end{array}$ & $\begin{array}{l}\mathbf{B} \text { (i) } \\
\mathbf{B} \text { (ii) } \\
\mathbf{B} \text { (iii) } \\
\mathbf{B} \text { (iv) }\end{array}$ & $\begin{array}{c}\text { T.P. } \\
\text {," } \\
,,\end{array}$ & $\begin{array}{l}18 \\
29 \\
44 \\
23\end{array}$ & $\begin{array}{l}32 \\
34 \\
26 \\
33\end{array}$ & $\begin{array}{l}34 \\
27 \\
20 \\
30\end{array}$ & $\begin{array}{l}16 \\
10 \\
10 \\
14\end{array}$ \\
\hline $\begin{array}{l}\text { Rock dusts } \\
\text { K.G.F. dry drilling } \dagger \\
\text { K.G.F. wet ", }\end{array}$ & $\begin{array}{l}\text { C (i) } \\
\text { C (ii) }\end{array}$ & $\begin{array}{l}\text { T.P. } \\
\text {, }\end{array}$ & $\begin{array}{l}37 \\
47\end{array}$ & $\begin{array}{l}51 \\
43\end{array}$ & $\begin{array}{r}10 \\
8\end{array}$ & $\begin{array}{l}2 \\
2\end{array}$ \\
\hline $\begin{array}{l}\text { S.A. dry drilling } \ddagger \\
\text { S.A. wet } \quad, \quad \text {. }\end{array}$ & $\begin{array}{l}\mathrm{D} \text { (i) } \\
\mathrm{D} \text { (ii) }\end{array}$ & ", & $\begin{array}{l}16 \\
82\end{array}$ & $\begin{array}{l}43 \\
13\end{array}$ & $\begin{array}{r}26 \\
4\end{array}$ & $\begin{array}{r}15 \\
1\end{array}$ \\
\hline
\end{tabular}

*Samples in group (A) were taken with a gravimetric sampler (Watson and Morris, 1952). Portions of these dusts were dispersed in Canada balsam for size distributions to be made. Thus, aggregates in Canada balsam for size distributions to be made. Thus, aggregates
were broken down, in contrast with the assessment of the T.P. samples for which aggregates were counted and sized as single particles. †K.G.F. = samples taken in the mines of the Kolar Gold Field in India (Watson 1952). $\$$ S.A. = samples taken in the goldmines of South Africa (Patterson, 1939-40). Three of the filter samples appear to be deficient in small particles. This is probably a function of the mounting technique. For the purpose of the present argument the distorted size-distributions thus obtained are of value in that they extend the range considered.

The first point to note concerning these various dusts is that they cover a very wide range of size distributions, in both the coal and the rock dust series. The two series contain dusts of different densities, approximately $1.3 \mathrm{~g} / \mathrm{cc}$. for the coal dusts and approximately 2.65 for the rock dusts.

For 100 particles of each dust, values of the following functions have been calculated :-

$$
\begin{array}{llll}
\text { For number } & \ldots & \Sigma(n . r) \\
\text { For area } & \ldots & \Sigma\left(\mathrm{nd}^{2} . r\right) & \text { Equation (3) } \\
\text { For volume } & \ldots & \Sigma\left(\mathrm{nd}^{3} . \mathrm{r}\right) &
\end{array}
$$

where $\mathrm{n}$ is the number of particles in a small size group of mean diameter $\mathrm{d}$, and $\mathrm{r}$ is the fractional lung deposition or selector device penetration at this mean size. In all these calculations the mean projected diameter has been used as a measure of particle size, the curves for the selector device and for lung deposition being appropriate to the density and value of $d_{p} / d_{s}$ of the dust.

In Table 4 are given the ratios of the values of those functions for number, area, and mass (or volume) for (1) penetration through standard elutriator or horizontal separation to lung deposi-
TABLE 4

CALCUlated VALUE OF RATIOS OF PENETRATION THROUGH SEPARATING INSTRUMENTS TO LUNG

\begin{tabular}{|c|c|c|c|c|c|c|}
\hline \multirow{3}{*}{$\begin{array}{l}\text { Dust } \\
\text { Code } \\
\text { No. }\end{array}$} & \multirow{2}{*}{\multicolumn{3}{|c|}{$\begin{array}{c}\begin{array}{c}\text { Penetration through } \\
\text { Elutriator }\end{array} \\
\text { Lung Deposition }\end{array}$}} & \multirow{2}{*}{\multicolumn{3}{|c|}{$\begin{array}{c}\begin{array}{c}\text { Penetration through } \\
\text { Cyclone }\end{array} \\
\text { Lung Deposition }\end{array}$}} \\
\hline & & & & & & \\
\hline & Number & Area & Mass & Number & Area & Mass \\
\hline $\begin{array}{l}\text { A (i) } \\
\text { (ii) } \\
\text { (iii) } \\
\text { (iv) }\end{array}$ & $\begin{array}{l}2 \cdot 79 \\
2 \cdot 43 \\
2 \cdot 98 \\
2 \cdot 46\end{array}$ & $\begin{array}{l}4 \cdot 16 \\
3 \cdot 42 \\
4 \cdot 26 \\
4 \cdot 10\end{array}$ & $\begin{array}{l}3.75 \\
3.00 \\
3.73 \\
4.06\end{array}$ & $\begin{array}{l}2 \cdot 72 \\
2 \cdot 38 \\
2 \cdot 37 \\
2 \cdot 84\end{array}$ & $\begin{array}{l}3.62 \\
3.60 \\
3 \cdot 10 \\
3.55\end{array}$ & $\begin{array}{l}\cdot 37 \\
3 \cdot 42 \\
3 \cdot 06 \\
3 \cdot 27\end{array}$ \\
\hline $\begin{array}{l}\text { B (i) } \\
\quad \text { (ii) } \\
\text { (iii) } \\
\text { (iv) }\end{array}$ & $\begin{array}{l}2 \cdot 84 \\
2 \cdot 72 \\
2 \cdot 75 \\
2 \cdot 77\end{array}$ & $\begin{array}{l}4 \cdot 30 \\
4 \cdot 11 \\
4 \cdot 18 \\
4 \cdot 04\end{array}$ & $\begin{array}{l}3.99 \\
4.02 \\
4.05 \\
3.62\end{array}$ & $\begin{array}{l}2 \cdot 66 \\
2 \cdot 57 \\
2 \cdot 57 \\
2 \cdot 59\end{array}$ & $\begin{array}{l}3 \cdot 44 \\
3 \cdot 16 \\
3 \cdot 38 \\
3 \cdot 33\end{array}$ & $\begin{array}{l}3 \cdot 32 \\
3 \cdot 32 \\
3 \cdot 36 \\
3 \cdot 14\end{array}$ \\
\hline C (i) & $\begin{array}{l}2 \cdot 88 \\
2 \cdot 32\end{array}$ & $\begin{array}{l}3 \cdot 33 \\
3 \cdot 30\end{array}$ & $\begin{array}{l}3.96 \\
3.89\end{array}$ & $\begin{array}{l}2 \cdot 19 \\
2 \cdot 21\end{array}$ & $\begin{array}{l}2.92 \\
2.87\end{array}$ & $\begin{array}{l}3 \cdot 30 \\
3 \cdot 17\end{array}$ \\
\hline $\begin{array}{l}\text { D (i) } \\
\text { (ii) }\end{array}$ & $\begin{array}{l}2 \cdot 56 \\
2 \cdot 52\end{array}$ & $\begin{array}{l}3.68 \\
3.61\end{array}$ & $\begin{array}{l}3 \cdot 19 \\
3 \cdot 57\end{array}$ & $\begin{array}{l}2 \cdot 42 \\
2 \cdot 38\end{array}$ & $\begin{array}{l}3.35 \\
2.75\end{array}$ & $\begin{array}{l}3.47 \\
2.55\end{array}$ \\
\hline Means & $2 \cdot 62$ & 3.87 & 3.74 & 2.49 & $3 \cdot 26$ & $3 \cdot 23$ \\
\hline S.D. & 0.22 & 0.379 & 0.344 & 0.20 & 0.294 & 0.244 \\
\hline
\end{tabular}
DEPOSITION FOR A NUMBER OF DUSTS IN TERM OF NUMBER, SURFACE AREA, AND MASS

tion, and (2) penetration through cyclone (Dust 1) to lung deposition.

It must be pointed out that many simplifying assumptions have been made in producing the figures in Table 4. The main ones are that the experimental results given by Brown and others (1950) are reliable. The china clay dust used by Brown and others was similar in physical characteristics to the sample examined to determine the shape factors used to standardize the experimental lung deposition curve. The statistical values of the ratios $d_{p}$ to $d_{s}$ for coal and quartz are reliable. The limiting size of particles that can reach the alveoli is correct, i.e., a sphere of unit density and $12 \mu$ diameter. In making the calculations for the elutriator it has been assumed that the theoretical relationship of Equation (2) is achieved in practice.

The results of Table 4 give some of the properties of selectors in comparison with the lung deposition curves. For example, in comparison with the standardized lung deposition of a dust cloud, the amount calculated to pass either of the selectors, and hence collected as the sample, is comparatively insensitive to particle size distribution, shape, or density. The relative amount of dust calculated to pass the selector of either kind is similar in terms of number, area, and mass respectively. For comparative assessment of the physical concentration of the respirable fraction of a dust, either selector considered would be suitable, irrespective of the unit of concentration. An absolute measure could be derived by dividing the practical value by the 
appropriate mean ratio given at the foot of Table 4. More dust is actually included in the sample than would be deposited in the lung when breathing at the same minute volume as the sampling rate. As the size-distribution of the dust passing either selector is not the same as that reaching the lung the chemical and petrological composition of the actual sample may be a little different from the dust actually reaching the lung, when there is a marked change in composition with particle size. Thus it should be possible to use with confidence selective sampling devices to simulate the human lung. The best units in which to express dust concentration is a matter for further investigation, but there are advantages in deciding on mass, for it is easily determined with precision using standard apparatus. For comparative results to be obtained in different situations it is necessary, however, to standardize the design of sampling apparatus. Further, it is important that the sampling rate be held constant at the designed value, using, for instance, a critical orifice. For, if it changes, the particle-size/selection curve changes too, and the error in the amount of sample collected will be about double what it would be without the selector device.

Naturally, the above refers to a "standard" lung. The actual amount of dust deposited in any instance will depend on breathing rate and breathing pattern (Davies, 1952).

\section{Summary}

An examination is made of the possibilities of designing a dust-sampling instrument to give directly an index of concentration proportional to the amount of dust likely to be deposited in the lung. Such deposition will depend, among other things, on the size-distribution of the dust particles in the cloud being breathed, and on their density and shape. The aerodynamic diameter of a particle is the correct measure of its size in this context, and results are given for a number of dusts of the relation between this and the mean projected diameter as determined with a microscope.

Lung deposition versus particle size curves are derived for a number of natural dusts. These are compared with the curves of penetration of dust through some selector devices which trap the larger particles, leaving the smaller ones to pass through to constitute the sample.

The results obtained suggest strongly that it is possible to use with confidence selective sampling devices which satisfactorily simulate the upper passages of the human respiratory system, and will allow to pass to a suitable depositing element a sample from which can be derived directly a measure of the concentration of the respirable fraction of a dust. This sample will give a comparable index of concentration which is almost independent of the dust, its size-distribution, density, and particle shape.

\section{REFERENCES}

Brown, J. H., Cook, K. M., Ney, F. G., and Hatch, T. (1950). Amer. J. publ. Hlth., $40,450$.

Davies, C. N. (1949). British Journal of Industrial Medicine, 6, 245. Davies, C. N. (1949). 120 .

--(1952). Ibid.,9,120. Engineering, Lond., 167, 165.

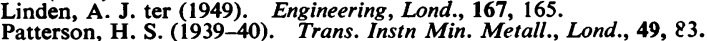

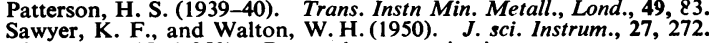
Walton, W. H. (1950). Personal communication.

(1952). Nature, Lond., 169, 518.

Watson, H. H. (1952). Trans. Instn Min. Metall., Lond., 61, 185.

Wilson. and Morris, T. G. (1952). Engineering, Lond., 174, 228. $30,265$. 